Sharif University of Technology
Scientia Iranica
Transactions E: Industrial Engineering
hCIENTIA

\title{
Investigation into skill leveled operators in a multi-period cellular manufacturing system with the existence of multi-functional machines
}

\author{
M. Rafiee* and A. Mohamaditalab \\ Department of Industrial Engineering, Sharif University of Technology, Tehran, Iran. \\ Received 3 June 2017; received in revised form 3 July 2018; accepted 21 July 2019
}

\author{
KEYWORDS \\ Multi-period cellular \\ manufacturing \\ system; \\ Machine reliability; \\ Workforce learning- \\ forgetting effect; \\ Alternative process \\ routing.
}

\begin{abstract}
Numerous studies published in the field of cellular manufacturing system are based on the assumption that machines are reliable in the entire production line without any breakdown. Since such assumptions are not usually realistic, to contribute to closing this gap between assumption and reality, a new model was proposed that additionally considered machine reliability, alternative process routings, and workforce assignment in a dynamic environment. Given such considerations in this research, the modified problem was defined and formulated and an extended mixed integer multi-period mathematical model was proposed. In order to evaluate the effectiveness and capability of the extended model, some hypothetical numerical instances were generated and computational experiments were carried out using GAMS optimization package. Experimental results demonstrated that the demand value could affect the machine breakdown rate, and a machine with a minimum breakdown rate was implemented more often than others. Moreover, the observed tradeoff between the workforce-related costs and cell-formation costs indicated that workforcerelated issues had a significant impact on the total efficiency of the system. The proposed model can be quite applicable to medium- and large-scale manufacturing companies.

(C) 2020 Sharif University of Technology. All rights reserved.
\end{abstract}

\section{Introduction}

Group Technology (GT), known as an effective manufacturing technique, necessitates, as the name suggests, fulfilling similar tasks in the same way. This approach can be viably employed in a competitive production environment, which makes manufacturing systems adapt themselves to the erratic changes and dynamics of production factors such as part demand changes, new product development, machine requirements, etc. Being highly potential and enjoying high performance in

*. Corresponding author. Tel.: +98 2166165725 E-mail addresses: rafiee@sharif.edu (M. Rafiee); mohamaditalab88@gmail.com (A. Mohamaditalab)

doi: $10.24200 /$ sci. 2019.21513 manufacturing, Cellular Manufacturing System (CMS) as an application of GT can be implemented at most industrial plants. However, Cell Formation (CF), Inter/intra-Cell Layout (CL), and workforce allocation are the three main sub-problems in designing an efficient CMS. Many researcher have tackled these problems effectively, especially in case of complicated models, in which two or three of the abovementioned problems are simultaneously taken into account. Liu et al. [1], for instance, presented a new model by integrating production planning with facility transfer in a dynamic cellular manufacturing environment in the supply chain. Similarly, Askin [2] reviewed the development of CMS-related organizational issues and options. Accordingly, relevant studies can be categorized in terms of designing and optimization. Among other studies, Ameli and Arkat [3] aimed to solve the 
CF problem and developed a pure integer mathematical model. They also considered issues of machine reliability and Alternative Process Routings (APRs). Bulgak and Bektas [4] conducted another research in which $\mathrm{CF}$ problem along with Production Planning was investigated, and reconfiguring the system was simultaneously taken into consideration. Mehdizadeh et al. [5] presented a multi-objective Mixed Integer Programming (MIP) model to simultaneously solve CF and production planning problems. They considered numerous real-world parameters such as alternative plans for processing the part types, flexibility of workers and machines, multi-period production planning, capacity of machines, reconfiguration of dynamic systems, sequence of operations, duplicate machines, time availability of workers, and worker assignment were taken into consideration. Furthermore, Mahdavi et al. [6] extended a dynamic CF considering PP and workforce assignment which aimed to minimize the current inventory, in-process inventory and backlog, inter-cell part trip, reconfiguration of machines, and workforcerelated costs. Similarly, Aryanezhad et al. [7] proposed an extended model to address CF and workforce assignment problems while, at the same time, examining the following real-world production factors: flexibility of part routings, machine, and labor enhancement training for mastery of higher skill level. Similarly, Bagheri and Bashiri [8] proposed a comprehensive model in which the dynamic $\mathrm{CF}$ problems, including inter-cell layout and workforce assignment problems, were integrated. In fact, in a dynamic environment, they analyzed the learning ability of labors. Javadi et al. [9] introduced a novel model in order to investigate the layout and CF problems simultaneously. Their proposed model attempted to design the materialhandling flow path structure and inter/intra-cell layout problem concurrently. Bagheri et al. [10] considered a newfangled model for CMS considering some productoin features comprising reliability of machines with stochastic parameters such as service and arrival times in a dynamic area and APRs. Moreover, they employed a benders decomposition method to overcome the complexity of the mentioned problem. Bayram and Sahin [11] also proposed a mathematical model in which many real-world production factors such as sequences of operations, splitting of lots, changing demands for products, capacities of machines, the products' alternative processing routes, and machine duplication were addressed.

Nowadays, in competitive production systems, the workforce-related issues are of importance. Therefore, it is necessary to review the relevant studies in this domain. Recently, Azadeh et al. [12] applied a novel model to the dynamic CMS in a multiobjective area called MDCMS with emphasis on human factors. Two main objectives including minimizing the inconsistency of the decision-making mode for the workforce in the public manufacturing cells and balancing the workload of the cells with respect to workforce efficacy were emphasized in their research. Moreover, in their research, Liu et al. [13] aimed to develop a combined decision model of production planning and assignment of workers in a dynamic CMS area of fiber connector manufacturing business. In the same manner, Mehdizadeh and Rahimi [14] attempted to develop a joint model to tackle the dynamic CF problem with emphasis on the assignment of workforce and inter/intra cell layout problems in the presence of machine duplication. Similarly, Rafiei and Ghodsi [15] proposed an ant colony optimization to tackle the problem of CF integrated with workforce-related issues. Sakhaii et al. [16] also proposed and applied robust optimization methods to investigate the dynamic CF problem with focus on the concepts of reliability of machines, production planning, allocation of workforce, inter cell layout, and APRs. A comprehensive multiobjective model of the CMS in a dynamic area was extended by Nouri [17] who considered several key cell design factors including designations of machines, inter/intra-cell material handling, allotting of workers, workload balancing and outsourcing according to the operation time, and the operation sequence of parts. However, in this study and other related researches, workforce-related issues were not addressed in detail. In addition, the present study aimed to fill the gap mentioned earlier.

In order to analyze the problem in detail, the most significant factors affecting CMS performance are listed in Table 1.

Table 1. Essential parameters of a Cellular Manufacturing System (CMS) problem.

\begin{tabular}{ll}
\hline Factor code & \multicolumn{1}{c}{ Factor description } \\
\hline 1 & Process sequence \\
2 & Part trips \\
$2-\mathrm{A}$ & Inter-cell part trip \\
$2-\mathrm{B}$ & Intra-cell part trip \\
3 & Cell formation \\
4 & Machine breakdown \\
5 & Process time/cost \\
6 & Machine time/process capacity \\
7 & Multi-functional machines \\
8 & Cell load variation \\
9 & Operator related issues \\
$9-\mathrm{A}$ & Hiring-firing \\
$9-\mathrm{B}$ & Salary \\
$9-\mathrm{C}$ & Training \\
$9-\mathrm{D}$ & Operator inter-intra cell trips \\
$9-\mathrm{E}$ & Skill level \\
10 & Uncertainty \\
\hline
\end{tabular}


Based on the factors listed in Table 1, it has been attempted to analyze the recent studies, the obtained results of which are reported in Table 2. According to this table, several vital realistic assumptions such as reliability of machines, APRs, and workforce learningforgetting effect have been neglected by a number of previous studies. In the following, however, an optimization problem will be introduced and generalized by emphasizing the APRs and workforce-related issues while multi-functional machines are available and machines are not reliable. The generalized problem is presented with the aim of reducing the inter-cell part trips and minimizing machine breakdown and workforce-related costs. In fact, the proposed model is an extended version of the research conducted by Bagheri and Bashiri [8], to which many other realistic factors such as APRs, machine reliability, and workforce learning-forgetting effect are added.

In the following, a Mixed Integer Non-Linear Mathematical Programming (MINLP) model will be proposed which is in line with the mentioned objectives. Then, a linearization technique was implemented to convert the model into an MIP form. Section 3 analyzes the effectiveness of the presented model which is verified through giving some numerical examples followed by the conclusion and some suggestions for future research in the last section.

\section{The optimization model}

\subsection{Problem explanation and mathematical formulation}

A majority of the previous approaches are based on an unrealistic assumption that machines are reliable in the whole production horizon without any breakdown. In fact, in industrial environments, machines are unreliable and their breakdown costs should be considered in order to enhance the efficiency of CMS. To this end, this paper proposes a framework to consider the costs of machine breakdown, i.e., repairing and installation-uninstallation costs. Consequently, exponential distribution should be considered with a given breakdown (failure) rate of machine reliability in its operating time:

$$
R=\exp (-\lambda t)
$$

where $R$ is the machine reliability at time $t$. The breakdown rate $\lambda$ is also given in the planning horizon; therefore, the mean time among the failures called MTBF is determined through Eq. (2):

$$
M T B F=\frac{1}{\lambda} \text {. }
$$

To determine the total machine breakdown cost within its production horizon, the total production time is divided by its MTBF and then, the obtained value is multiplied by machine-failure unit cost.
Other basic assumptions considered in modeling the problem are described as follows:

1. Some features are already given and fixed over the planning horizon such as the number of cells, demand of each part type in each period, and lower/upper bounds of cell capacity;

2. There are machine tools that can be installed on the predefined machines. Each tool can be used to machine a specific operation of a part;

3. Workforce can be assigned to the responsibility of more than one tool or machine according to their skill level;

4. Training the operators is allowed; in other words, workforce could be taught to work with a particular machine by paying a teaching cost. However, the trained workforce can be applied in other periods without any extra training cost. Besides, according to a predefined forgetting rate, workforce may forget working with a tool.

\subsection{Notations}

\section{Indices:}

$g \quad$ Machine tools, $g^{\prime}=1, \ldots, G$

$i=1, \ldots, I \quad$ The number of parts

c The number of machine cells that should be constructed, $c^{\prime}=1, \ldots, C$

$j=1, \ldots, O \quad$ The number of operations for each part type

$t=1, \ldots, T \quad$ The number of manufacturing cycles (term)

$k=1, \ldots, K$ The number of available workforce

$l=1, \ldots, L \quad$ Workforce skill level

\section{Input parameters:}

$M C_{i} \quad$ Inter-cell part trip cost

$S M \quad$ Machine install/uninstall cost in a cell

$S G \quad$ Tool install/uninstall cost on a machine

$\Psi_{i j g} \quad$ Tool consumption cost

$B_{m} \quad$ The repairing cost of machine " $m$ "

mcaptime $_{m}$ The maximum time of processing by machine " $m$ "

$u_{m}, l_{m} \quad$ The maximum and minimum numbers of tools that can be installed on machine " $m$ "

$u_{c}, l_{c} \quad$ The maximum and minimum numbers of machines that could be assigned to cell " $c$ "

$q \quad$ The percentage of cell load variation

$M T B F=\frac{1}{\lambda_{m}}$ The average time of the machine " $m$ " 
Table 2. Literature review

\begin{tabular}{|c|c|c|c|c|c|c|c|c|c|c|c|c|c|c|c|c|}
\hline \multirow{3}{*}{$\begin{array}{l}\text { Author's name } \\
\text { Bayram and Şahin [10] }\end{array}$} & \multirow{3}{*}{$\begin{array}{l}\text { Year } \\
2016\end{array}$} & \multirow{3}{*}{$\begin{array}{l}\text { F1 } \\
*\end{array}$} & \multicolumn{2}{|c|}{ F2 } & \multirow[t]{2}{*}{ F3 } & \multirow[t]{2}{*}{ F4 } & \multirow[t]{2}{*}{ F5 } & \multirow[t]{2}{*}{ F6 1} & \multirow[t]{2}{*}{ F7 1} & \multirow[t]{2}{*}{ F8 } & \multicolumn{5}{|c|}{ F9 } & \multirow[t]{2}{*}{ F10 } \\
\hline & & & F 2- & $2-\mathrm{A}$ & & & & & & & \multicolumn{5}{|c|}{ F9-E F9-D F9-C F9-B F9-A } & \\
\hline & & & - & $*$ & $*$ & - & - & - & - & $*$ & - & - & - & - & - & - \\
\hline Nouri [16] & 2016 & & * & $*$ & $*$ & - & - & - & - & $*$ & - & - & - & - & $*$ & - \\
\hline Liu, et al. [12] & 2016 & $*$ & - & - & $*$ & - & - & $*$ & - & - & $*$ & - & $*$ & - & - & - \\
\hline Deep and Singh [17] & 2015 & $*$ & $*$ & $*$ & $*$ & - & $*$ & $*$ & - & - & - & - & - & - & - & - \\
\hline Renna and Ambrico [18] & 2015 & $*$ & - & $*$ & $*$ & - & $*$ & $*$ & - & - & - & - & - & - & - & $*$ \\
\hline Esmailnezhad, et al. [19] & 2015 & $*$ & - & - & - & $*$ & $*$ & & - & - & - & - & - & - & - & $*$ \\
\hline Wu and Suzuki [20] & 2015 & $*$ & - & $*$ & - & - & $*$ & $*$ & & $*$ & - & - & - & - & - & - \\
\hline Alhourani [21] & 2015 & $*$ & $*$ & $*$ & - & $*$ & $*$ & $*$ & - & - & - & - & - & - & - & - \\
\hline Ulutas [22] & 2015 & $*$ & - & & - & - & $*$ & & - & - & - & - & - & - & - & - \\
\hline Nouri[23] & 2015 & $*$ & * & $*$ & - & - & $*$ & - & - & $*$ & - & $*$ & - & $*$ & $*$ & - \\
\hline Kumar and Sharma [24] & 2015 & $*$ & - & $*$ & - & $*$ & - & $*$ & - & - & - & - & - & - & - & - \\
\hline Sakhaii, et al. [25] & 2015 & $*$ & $*$ & $*$ & $*$ & $*$ & - & & - & - & - & - & $*$ & & $*$ & $*$ \\
\hline Niakan, et al. [26] & 2015 & $*$ & * & $*$ & * & - & $*$ & $*$ & - & - & - & - & - & * & * & $*$ \\
\hline Erozan, et al. [27] & 2015 & $*$ & - & - & - & - & $*$ & - & - & $*$ & - & - & - & - & - & - \\
\hline Brusco [28] & 2015 & $*$ & - & - & - & - & - & - & - & - & - & - & - & - & - & - \\
\hline Won and Logendran [29] & 2015 & $*$ & - & - & - & - & $*$ & $*$ & - & $*$ & - & - & - & - & - & - \\
\hline Halat and & 2015 & $*$ & - & $*$ & - & - & $*$ & - & - & - & - & - & - & - & - & - \\
\hline Bashirzadeh [30] & & & & & & & & & & & & & & & & \\
\hline Yadollahi, et al. [31] & 2014 & $*$ & * & $*$ & & $*$ & - & $*$ & - & - & - & - & - & - & - & - \\
\hline Egilmez, et al. [32] & 2014 & $*$ & - & - & - & - & $*$ & - & - & - & - & - & - & - & - & $*$ \\
\hline Park, et al. [33] & 2014 & & - & - & - & - & - & - & - & - & - & $*$ & - & - & - & - \\
\hline Sharifi, et al. [34] & 2014 & $*$ & - & $*$ & $*$ & - & $*$ & $*$ & - & - & - & - & - & - & - & - \\
\hline Baykasoglu and & 2014 & * & - & - & - & - & - & - & - & - & - & - & - & - & - & - \\
\hline Gorkemli [35] & & & & & & & & & & & & & & & & \\
\hline Mohammadi and & 2014 & $*$ & $*$ & * & - & - & $*$ & * & - & - & - & - & - & - & - & - \\
\hline Forghani [36] & & & & & & & & & & & & & & & & \\
\hline Bootaki, et al [37] & 2014 & $*$ & - & - & - & - & - & - & - & - & & $*$ & - & - & - & - \\
\hline Jabal-Ameli and & 2014 & $*$ & - & - & - & - & - & - & - & - & - & - & - & - & - & - \\
\hline Moshref-Javadi [28] & & & & & & & & & & & & & & & & \\
\hline Raja and Anbumalar [39] & 2014 & $*$ & - & $*$ & - & - & - & - & - & - & - & - & - & - & - & - \\
\hline Kao and Chen [40] & 2014 & $*$ & - & - & - & - & - & - & $*$ & - & - & - & - & - & - & - \\
\hline This paper & 2016 & $*$ & $*$ & $*$ & $*$ & $*$ & $*$ & $*$ & $*$ & $*$ & $*$ & * & $*$ & $*$ & $*$ & - \\
\hline
\end{tabular}




\begin{abstract}
$H, F$
Workforce hiring/firing cost

$S A_{l} \quad$ The salary of the workforce with the skill level $l$

movek The workforce trip cost of cells

$a_{k g}^{t=1} \quad 1$ if workforce $k$ can work with tool $g$ at the start time of planning horizon

$a w_{g} \quad$ The minimum skill level to work with tool " $g$ "

$\partial_{l} \quad$ Skill level boundary

$w_{1}, w_{2} \quad$ Increase and decrease in workforce skill level

$D_{i}^{t} \quad$ The demand value for the $i$-th part in the $t$-th manufacturing term

$d i s_{c c^{\prime}} \quad$ The distance between the two

time tijgm $^{\text {The processing time of operation } j \text { on }}$ machine type $m$ for part type $i$ with tool $g$

MINEM The least number of workforce sufficient to be hired in each manufacturing term

Opcaptime The maximum time of working for each workforce

$\alpha_{i j g}^{t} \quad 1$ if operation $j$ of part type $i$ can be processed by tool $g$ in production period $t ; 0$ otherwise

$\beta_{g m}^{t} \quad 1$ if tool $g$ can be installed on machine $m$ in production period $t ; 0$ otherwise
\end{abstract}

\section{Decision variables:}

$x_{m c}^{t}= \begin{cases}1 ; & \begin{array}{l}\text { If machine } m \text { in period } t \text { is designated } \\ \text { to cell } c\end{array} \\ 0 ; & \text { Otherwise }\end{cases}$

$y_{g m}^{t}= \begin{cases}1 ; & \text { If tool } g \text { in period } h \text { is installed } \\ & \text { on machine } m \\ 0 ; & \text { Otherwise }\end{cases}$

$p_{i j g}^{t}= \begin{cases}1 ; & \begin{array}{l}\text { If operation } j \text { of part type } i \text { in period } t \\ \text { is processed with tool } g\end{array} \\ 0 ; & \text { Otherwise }\end{cases}$

$b_{k}^{t}= \begin{cases}1 ; & \text { If operator } k \text { is working in period } t \\ 0 ; & \text { Otherwise }\end{cases}$

$h_{k}^{t}= \begin{cases}1 ; & \text { If operator } k \text { is hired in period } t \\ 0 ; & \text { Otherwise }\end{cases}$

$w_{k g}^{t}= \begin{cases}1 ; & \text { If operator } k \text { is working with machine } \\ & \text { tool } g \text { in period } t \\ 0 ; & \text { Otherwise }\end{cases}$

$$
\begin{aligned}
& l e_{k l}^{t}= \begin{cases}1 ; & \text { If operator } k \text { is in skill level of } l \\
\text { in period } t & \end{cases} \\
& a_{k g}^{t \geq 2}= \begin{cases}1 ; & \text { Otherwise }\end{cases} \\
& 0 ; \quad \text { ifoperator } k \text { can woriod } t
\end{aligned}
$$

\subsection{The objective function}

The proposed MINLP model for the CMS design is offered as Eqs. (3) to (11):

min Model 1:

$$
\begin{aligned}
& \sum_{t=1}^{T} \sum_{i=1}^{I} \sum_{j=1}^{O_{i}} \sum_{g, g^{\prime}=1}^{G} \sum_{m, m^{\prime}=1}^{M} \sum_{c, c \neq c^{\prime}} x_{m c}^{t} \times y_{g m}^{t} \times p_{i j g}^{t} \times x_{m^{\prime} c^{\prime}}^{t} \\
& \times y_{g^{\prime} m^{\prime}}^{t} \times p_{i(j+1) g^{\prime}}^{t} \times D_{i}^{t} \times d i s_{c, c^{\prime}} \times M C_{i}, \\
& +\sum_{t=1}^{T} \sum_{i=1}^{I} \sum_{j=1}^{O_{i}} \sum_{g, g^{\prime}=1}^{G} \sum_{c=1}^{C} \sum_{m, m^{\prime} \neq m} x_{m c}^{t} \times y_{g m}^{t} \times p_{i j g}^{t} \\
& \times x_{m^{\prime} c}^{t} \times y_{g^{\prime} m^{\prime}}^{t} \times p_{i(j+1) g^{\prime}}^{t} \times D_{i}^{t} \times M C_{i}, \\
& +\sum_{t=1}^{T-1} \sum_{m=1}^{M} \sum_{c, c \neq c^{\prime}} x_{m c}^{t} \times x_{m c^{\prime}}^{t+1} \times d i s_{c, c^{\prime}} \times S M, \\
& +\sum_{t=1}^{T} \sum_{m=1}^{M} \sum_{i=1}^{I} \sum_{j=1}^{O_{i}} \sum_{g=1}^{G} y_{g m}^{t} \times p_{i j g}^{t} \times D_{i}^{t} \times \Psi_{i j g} \\
& +\sum_{t=1}^{T-1} \sum_{g=1}^{G} \sum_{m, m \neq m^{\prime}} y_{g m}^{t} \times y_{g m^{\prime}}^{t+1} \times S G, \\
& +\sum_{t=1}^{T} \sum_{m=1}^{M} \frac{\sum_{i=1}^{I} \sum_{j=1}^{O_{i}} \sum_{g=1}^{G} p_{i j g}^{t} \times y_{g m}^{t} \times t i m e_{i j g m} \times D_{i}^{t}}{M T B F_{m}} \\
& \times B_{m}, \\
& \sum_{k=1}^{K}\left(h_{k}^{1} \times H\right)+\sum_{t=2}^{T} \sum_{k=1}^{K}\left(h_{k}^{t} \times H+\left(1-b_{k}^{t}\right) \times F\right), \\
& +\sum_{t=1}^{T} \sum_{k=1}^{K} \sum_{g, g^{\prime}} \sum_{m, m^{\prime}} \sum_{c, c \neq c^{\prime}} w_{k g}^{t} \times w_{k g^{\prime}}^{t} \times y_{g m}^{t} \times x_{m c}^{t} \\
& \times y_{g^{\prime} m^{\prime}}^{t} \times x_{m^{\prime} c^{\prime}}^{t} \times d i s_{c, c^{\prime}} \times \text { movek, } \\
& +\sum_{t=1}^{T} \sum_{k=1}^{K} \sum_{l=1}^{L} \sum_{g=1}^{G} \sum_{i=1}^{I} \sum_{j=1}^{O_{i}} \sum_{m=1}^{M} \sum_{c=1}^{C} p_{i j g}^{t} \times y_{g m}^{t}
\end{aligned}
$$


$\times x_{m c}^{t} \times t i m e_{i j g m} \times D_{i}^{t} \times w_{k g}^{t} \times l e_{l k}^{t} \times S A_{l}$,

Subjected to:

$\sum_{c=1}^{C} X_{m c}^{t}=1 \quad \forall m, t$

$\sum_{g=1}^{G} P_{i j g}^{t}=1 \quad \forall i, j, t$

$\sum_{m=1}^{M} Y_{g m}^{t} \leq 1 \quad \forall g, m, t ;$

$P_{i j g}^{t} \leq \alpha_{i j g}^{t} \quad \forall i, j, g, t$

$Y_{g m}^{t} \leq \beta_{g m}^{t} \quad \forall g, m, t$

$\sum_{c=1}^{C} \sum_{m=1}^{M} Y_{g m}^{t} \times X_{m c}^{t} \leq \sum_{i=1}^{I} \sum_{j=1}^{O_{i}} P_{i j g}^{t} \quad \forall g, t ;$

$\sum_{c=1}^{C} \sum_{m=1}^{M} Y_{g m}^{t} \times X_{m c}^{t} \geq P_{i j g}^{t} \quad \forall i, j, g, t ;$

$\sum_{m=1}^{M} X_{m c}^{t} \leq u_{c} \quad \forall c, t$

$\sum_{m=1}^{M} X_{m c}^{t} \geq l_{c} \quad \forall c, t$

$\sum_{g=1}^{G} Y_{g m}^{t} \leq u_{m} \quad \forall m, t$

$\sum_{g=1}^{G} Y_{g m}^{t} \geq l_{m} \quad \forall m, t$

$\sum_{i=1}^{I} \sum_{j=1}^{O_{i}} \sum_{g=1}^{G} P_{i j g}^{t} \times Y_{g m}^{t} \times$ time $_{i j g m} \times D_{i}^{t} \leq$ mcaptime

$\forall m, t$;

$$
\begin{gathered}
\sum_{i=1}^{I} \sum_{j=1}^{O_{i}} \sum_{g=1}^{G} X_{m c}^{t} \times Y_{g m}^{t} \times P_{i j g}^{t} \times \text { time }_{i j g m} \times D_{i}^{t} \\
\geq \frac{q}{C} \sum_{c=1}^{C} \sum_{i=1}^{I} \sum_{j=1}^{O_{i}} \sum_{g=1}^{G} X_{m c}^{t} \times Y_{g m}^{t} \times P_{i j g}^{t} \\
\quad \times t i m e_{i j g m} \times D_{i}^{t} \quad \forall c, t ; \\
b_{k}^{1}=h_{k}^{1} \quad \forall k ; \\
b_{k}^{t+1}\left(1-b_{k}^{t}\right)=h_{k}^{t+1} \quad \forall k, t ;
\end{gathered}
$$

$h_{k}^{t+1} \leq 1-b_{k}^{t} \quad \forall t=1, \ldots, T-1 \quad \forall k ;$

$$
\begin{aligned}
& \sum_{k=1}^{K} b_{k}^{t} \geq \min E M \quad \forall t \\
& w_{k g}^{t} \leq b_{k}^{t} \quad \forall k, g, t ; \\
& \sum_{k=1}^{K} w_{k g}^{t}=\sum_{m=1}^{M} y_{g m}^{t} \quad \forall g, t ;
\end{aligned}
$$

$\sum_{g=1}^{G} w_{k g}^{t} \geq b_{k}^{t} \quad \forall k, t$

$w_{k g}^{t} \times a w_{g} \leq a_{k g}^{t} \quad \forall k, g, t ;$

$a_{k g}^{t+1}=b_{k}^{t} \times a_{k g}^{t}+w_{k g}^{t} \times \omega_{1} \sum_{i=1}^{I} \sum_{j=1}^{O_{i}} \eta_{g}^{t}$

$+\left(1-b_{k}^{t}\right) \max \left(a_{k g}^{t}-\omega_{2}, 0\right) \quad \forall k, g t ;$

$\sum_{l=1}^{L-1} \partial_{l} \times l e_{(l+1) k}^{t} \leq \frac{\sum_{g=1}^{G} a_{k g}^{t}}{G} \leq \sum_{l=1}^{L} \partial_{l} \times l e_{l k}^{t} \quad \forall k, t ;$

$\sum_{l=1}^{L} l e_{l k}^{t}=1 \quad \forall k, t$

$\sum_{t=1}^{T} \sum_{g=1}^{G} \sum_{i=1}^{I} \sum_{j=1}^{O_{i}} \sum_{m=1}^{M} \sum_{c=1}^{C} p_{i j g}^{t} \times y_{g m}^{t} \times x_{m c}^{t} \times t i m e_{i j g m}$

$$
\times D_{i}^{t} \times w_{k g}^{t} \leq \text { opcaptime } \times b_{k}^{t} \quad \forall k, t ;
$$

$w_{k g}^{t}, h_{k}^{t}, b_{k}^{t}, l e_{k l}^{t} \in\{0,1\} \quad a_{k g}^{t} \geq 0$,

$X_{m c}^{t}, Y_{g m}^{t}, P_{i j g}^{t} \in\{0,1\}$.

The proposed model aims to minimize two main target groups: Group (1) includes the part and machine-related costs and Group (2) includes the workforce-related costs. The first term of the objective function, i.e., 3, aims to minimize the trips of inter-cell parts. Of note, an inter-cell part trip is determined based on inter-cell distances. The function of Part 4 is to minimize the intra-cell part trips. In addition, Part 5 minimizes the total cost of system reconfiguration. The dynamic nature of the production systems, i.e., demand, process routings, and machine characteristics variation, causes the machine to move among cells between two consecutive periods. This cost includes the uninstallation, movement, and installation of the machines among the cells. Part 6 minimizes the 
total consumption costs of tools. Moreover, Part 7 minimizes the installation/uninstallation costs of tools on different machines. Part 8 minimizes the overall machine breakdown cost. This cost is determined according to the total processing time of a machine and its breakdown rate. The function of Part 9 is to minimize the workforce hiring/firing costs. Part 10 takes control over the workforce among cells trips. Moreover, workforce salary cost is minimized using Part 11.

Constraint (12) guarantees that any type of machine is accurately assigned to a given cell. Eq. (13) ensures that the operation of each part can be performed by only one tool. It is assumed that a tool can be installed on only one machine. This constraint is guaranteed by Relation (14). Therefore, the presence of unused tools in a production period is possible. Relations (15) and (16) ensure that each operation and tool can be assigned to a tool and machine, respectively, with the capability of that installation. Constraints (17) and (18) ensure that the unused tool in a production period cannot be installed on any machine. The maximum and minimum numbers of machines for a cell are represented by Constraints (19) and (20), respectively, which are in the cell size range. Moreover, the allocated number of tools to each machine are presented by Constraints (21) and (22), respectively. The maximum amount of time that each machine consumes is eliminated by Constraint (23). Moreover, Constraint (24) balances the load variations of each cell during a production period. In the 1st period, if a worker is hired, he/she should be assigned to working with a machine. This issue is guaranteed by Constraint (25). The workforce hiring/firing balance between two consecutive periods is shown in Constraints (26) and (27). Constraint (28), in each manufacturing term, specifies the least number of workforce that should be hired. Workforce can work with a tool only if that workforce is implemented in a production period. Constraint (29) guarantees this point. Constraint 30 states that for a tool, workforce should be hired in a production period. Furthermore, if a workforce is hired, he/she should be assigned to some tools (machines) (Constraint (31)). Based on Constraint (32), workforce with a minimum skill value can be selected to work with a machine tool. The workforce learning-forgetting effect is assumed according to Constraints (33) and (34). Based on these two constraints, workforce skill level must be updated in each production period. Constraint (35) emphasizes that workforce should be ranked and given a skill level according to his/her abilities. Workforce time capacity for working in a production period is limited by Constraint (36). Finally, Constraints (37) defines the types of model variables.

\subsection{Linearization}

Since the presented MINLP model owing to the existence of nonlinearities in terms $3,4,5,6,7,8,10$, and 11 and Constraints (17), (18), (23), (24), (26), and (33) is a nonlinear one, here, it was transformed into a linear MIP model using three linearization techniques.

Proposition 1. Consider the pure quadratic 0-1 variable $Z=X_{1} \times X_{2} \times \ldots \times X_{n}$, where $X_{i}(i=1, \ldots, n)$ is a binary variable. The amount of variable $Z$ is obviously 1 if and only if all other variables are 1 ; otherwise 0 [8]. The mentioned mathematical view is formulated below by utilizing some new supplementary limitations.

$$
Z \leq X_{i} \quad \forall i=1, . ., n, \quad Z \geq \sum_{i=1}^{n} X_{i}-(n-1) .
$$

Proposition 2. Consider the variable $Z=X \times Y$ in which $X$ and $Y$ are binary and integer positive variables, respectively. Utilizing some new auxiliary constraints transforms the model into a linear form. The needed limitations are mentioned as follows:

$$
\begin{aligned}
& Z \leq M \times X ; \quad Z \leq Y ; \\
& Z \geq Y-(1-X) M ; \quad Z \geq 0 \text { and int. }
\end{aligned}
$$

Proposition 3. Consider the term:

$\min T$,

St :

$$
T=\max (X, a),
$$

where $X$ is a variable. By introducing some new auxiliary constraints, the mentioned nonlinear term could be converted into a linear one. The needed limitations are mentioned as follows:

$$
T \geq X, \quad T \geq a .
$$

Accordingly, new variables are defined as follows:

$$
\begin{aligned}
& P X Y 1_{i j g m c}^{t}=x_{m c}^{t} y_{g m}^{t} p_{i j g}^{t} x_{m^{\prime} c^{\prime}}^{t} y_{g^{\prime} m^{\prime}}^{t} p_{i(j+1) g^{\prime}}^{t}, \\
& P X Y 2_{i j g m c}^{t}=x_{m c}^{t} y_{g m}^{t} p_{i j g}^{t} x_{m^{\prime} c}^{t} y_{g^{\prime} m^{\prime}}^{t} p_{i(j+1) g^{\prime}}^{t}, \\
& X X_{m c c^{\prime}}^{t}=x_{m c}^{t} x_{m c^{\prime}}^{t+1}, \\
& Y X_{g m c}^{t}=y_{g m}^{t} x_{m c}^{t}, \\
& P Y_{i j g m}^{t}=p_{i j g}^{t} y_{g m}^{t}, \\
& P X Y_{i j g m c}^{t}=p_{i j g}^{t} x_{m c}^{t} y_{g m}^{t}, \\
& Y Y_{g m m^{\prime}}^{t}=y_{g m}^{t} y_{g m^{\prime}}^{t+1}, \\
& W W_{k g g^{\prime}}^{t}=w_{k g}^{t} w_{k g^{\prime}}^{t}, \\
& W L_{k g l}^{t}=w_{k g}^{t} l e_{l k}^{t},
\end{aligned}
$$




$$
\begin{aligned}
& B_{k}^{t}=b_{k}^{t+1} \times\left(1-b_{k}^{t}\right), \\
& Z=\max \left(a_{k g}^{t}-\omega_{2}, 0\right), \\
& B A_{k g}^{t}=b_{k}^{t} \times a_{k g}^{t}, \\
& B Z_{k}^{t}=\left(1-b_{k}^{t}\right) \times Z .
\end{aligned}
$$

The following supplementary limitations should be considered along with the previous model:

$$
\begin{aligned}
& P X Y 1_{i j g m c}^{t} \geq Y X_{g m c}^{t}+p_{i j g}^{t}+Y X_{g^{\prime} m^{\prime} c^{\prime}}^{t} \\
& +p_{i(j+1) g^{\prime}}^{t}-3, \\
& P X Y 1_{i j g m c}^{t} \leq Y X_{g m c}^{t}, \\
& P X Y 1_{i j g m c}^{t} \leq p_{i j g}^{t}, \\
& P X Y 1_{i j g m c}^{t} \leq Y X_{g^{\prime} m^{\prime} c^{\prime}}^{t}, \\
& P X Y 1_{i j g m c}^{t} \leq p_{i(j+1) g^{\prime}}^{t}, \\
& P X Y 2_{i j g m c}^{t} \geq Y X_{g m c}^{t}+p_{i j g}^{t}+Y X_{g^{\prime} m^{\prime} c}^{t} \\
& +p_{i(j+1) g^{\prime}}^{t}-3, \\
& P X Y 2_{\text {ijgmc }}^{t} \leq Y X_{g m c}^{t}, \\
& P X Y 2_{i j g m c}^{t} \leq p_{i j g}^{t}, \\
& P X Y 2_{i j g m c}^{t} \leq Y X_{g^{\prime} m^{\prime} c}^{t}, \\
& P X Y 2_{i j g m c}^{t} \leq p_{i(j+1) g^{\prime}}^{t}, \\
& X X_{m c c^{\prime}}^{t} \geq x_{m c}^{t}+x_{m c^{\prime}}^{t+1}-1, \\
& X X_{m c c^{\prime}}^{t} \leq x_{m c}^{t}, \\
& X X_{m c c^{\prime}}^{t} \leq x_{m c^{\prime}}^{t+1}, \\
& Y X_{g m c}^{t} \geq y_{g m}^{t}+x_{m c}^{t}-1, \\
& Y X_{g m c}^{t} \leq y_{g m}^{t}, \\
& Y X_{g m c}^{t} \leq x_{m c}^{t}, \\
& P Y_{i j g m}^{t} \geq p_{i j g}^{t}+y_{g m}^{t}-1, \\
& P Y_{i j g m}^{t} \leq p_{i j g}^{t}, \\
& P Y_{i j g m}^{t} \leq y_{g m}^{t}, \\
& P X Y_{i j g m}^{t} \geq x_{m c}^{t}+P Y_{i j g m}^{t}-1, \\
& P X Y_{i j g m}^{t} \leq P Y_{i j g m}^{t}, \\
& P X Y_{i j g m}^{t} \leq x_{m c}^{t},
\end{aligned}
$$

$$
\begin{aligned}
& W P X Y_{i j k g m}^{t} \leq w_{k g}^{t}+P X Y_{i j g m}^{t}-1, \\
& W P X Y_{i j k g m}^{t} \geq w_{k g}^{t}, \\
& W P X Y_{i j k g m}^{t} \geq P X Y_{i j g m}^{t}, \\
& Y Y_{g m m^{\prime}}^{t} \geq y_{g m}^{t}+y_{g m^{\prime}}^{t+1}-1,
\end{aligned}
$$

$Y Y_{g m m^{\prime}}^{t} \leq y_{g m}^{t}$,

$Y Y_{g m m^{\prime}}^{t} \leq y_{g m^{\prime}}^{t+1}$,

$W W_{k g g^{\prime}}^{t} \geq w_{k g}^{t}+w_{k g^{\prime}}^{t}-1$,

$W W_{k g g^{\prime}}^{t} \leq w_{k g}^{t}$,

$W W_{k g g^{\prime}}^{t} \leq w_{k g^{\prime}}^{t}$,

$W L_{k g l}^{t} \geq w_{k g}^{t}+l e_{l k}^{t}-1$,

$W L_{k g l}^{t} \leq w_{k g}^{t}$,

$W L_{k g l}^{t} \leq l e_{l k}^{t}$,

$W L P X Y_{i j k g m l}^{t} \geq W L_{k g l}^{t}+P X Y_{i j g m}^{t}-1$,

$W L P X Y_{i j k g m l}^{t} \leq W L_{k g l}^{t}$,

$$
W L P X Y_{i j k g m l}^{t} \leq P X Y_{i j g m}^{t},
$$

$W Y X_{k g g^{\prime} m m^{\prime} c c^{\prime}}^{t} \geq W W_{k g g^{\prime}}^{t}+Y Y_{g m m^{\prime}}^{t}$

$$
+X X_{m c c^{\prime}}^{t}-2,
$$




$$
B Z_{k}^{t} \leq M_{\infty} \times\left(1-b_{k}^{t}\right) .
$$

Therefore, the linear version of the MIP model can also be considered.

$\min$ Model $2=$

$$
\begin{aligned}
& \sum_{t=1}^{T} \sum_{i=1}^{I} \sum_{j=1}^{O_{i}} \sum_{g, g^{\prime}=1}^{G} \sum_{m, m^{\prime}=1}^{M} \sum_{c, c \neq c^{\prime}} \\
& P X Y 1_{i j g m c}^{t} \times D_{i}^{t} \times d i s_{c, c^{\prime}} \times M C_{i}, \\
& +\sum_{t=1}^{T} \sum_{i=1}^{I} \sum_{j=1}^{O_{i}} \sum_{g, g^{\prime}=1}^{G} \sum_{c=1}^{C} \sum_{m, m \neq m^{\prime}} P X Y 2_{i j g m c}^{t} \times D_{i}^{t}, \\
& +\sum_{t=1}^{T-1} \sum_{m=1}^{M} \sum_{c, c \neq c^{\prime}} X X_{m c c^{\prime}}^{t} \times d i s_{c, c^{\prime}} \times S M \\
& +\sum_{t=1}^{T} \sum_{m=1}^{M} \sum_{i=1}^{I} \sum_{j=1}^{O_{i}} \sum_{g=1}^{G} P Y_{i j g m}^{t} \times D_{i}^{t} \times \psi_{i j g}, \\
& +\sum_{t=1}^{T-1} \sum_{g=1}^{G} \sum_{m, m \neq m^{\prime}} Y Y_{g m m^{\prime}}^{t} \times S G, \\
& +\sum_{t=1}^{T} \sum_{m=1}^{M} \frac{\sum_{i=1}^{I} \sum_{j=1}^{O_{i}} \sum_{g=1}^{G} P Y_{i j g m}^{t} \times t i m e_{i j g m} \times D_{i}^{t}}{M T B F_{m}} \times B_{m} \\
& \sum_{k=1}^{K}\left(h_{k}^{1} \times H\right)+\sum_{t=2}^{T} \sum_{k=1}^{K}\left(h_{k}^{t} \times H+\left(1-b_{k}^{t}\right) \times F\right), \\
& \sum_{t=1}^{T} \sum_{k=1}^{K} \sum_{g, g^{\prime}} \sum_{m, m^{\prime}} \sum_{c, c^{\prime} \neq c} W Y X_{k g g^{\prime} m m^{\prime} c c^{\prime}}^{t} \times d i s_{c, c^{\prime}}, \\
& +\sum_{m=1}^{M} \sum_{t=1}^{T} \sum_{k=1}^{K} \sum_{l=1}^{L} \sum_{g=1}^{G} W L P X Y_{i j k g m l}^{t} \times t_{i m e_{i j g m}} \\
& \times D_{i}^{t} \times S A_{l} .
\end{aligned}
$$

The above model is conditional on the unaltered set of Constraints (12)-(17), (19)-(22), (25), (27)-32), (34), (35) and the new auxiliary Constraints (51)-(92).

Moreover, the set of Constraints (17), (18), (23), (24), (26), and (33) is replaced by:
$\sum_{c=1}^{C} \sum_{m=1}^{M} Y X_{g m c}^{t} \leq \sum_{i=1}^{I} \sum_{j=1}^{O_{i}} P_{i j g}^{t} \quad \forall g, t$

$\sum_{c=1}^{C} \sum_{m=1}^{M} Y X_{g m c}^{t} \geq P_{i j g}^{t} \quad \forall i, j, g, t$

$\sum_{i=1}^{I} \sum_{j=1}^{O_{i}} \sum_{g=1}^{G} P Y_{i j g m}^{t} \times$ time $_{i j g m} \times D_{i}^{t} \leq$ captime

$\forall m, t$;

$B_{k}^{t}=h_{k}^{t+1} \quad \forall k, t$

$a_{k g}^{t+1}=\omega_{1} \sum_{i=1}^{I} \sum_{j=1}^{O_{i}} W P X Y_{i j k g m}^{t}+B Z_{k}^{t}+B A_{k g}^{t}$

$\forall k, g, t$;

and the last set of constraint, i.e., Constraint (36) is replaced by:

$$
\begin{aligned}
& w_{k g}^{t}, h_{k}^{t}, b_{k}^{t}, l e_{k l}^{t}, B_{k}^{t}, p_{i j g}^{t}, W W_{k g g^{\prime}}^{t}, W L_{k g l}^{t}, x_{m c}^{t}, y_{g m}^{t}, \\
& P Y_{i j g m}^{t}, P X Y_{i j g m c}^{t}, P X Y 1_{i j g m c}^{t}, P X Y 2_{i j g m c}^{t}, \\
& W P X Y_{i j k g m}^{t}, W L P X Y_{i j k g m l}^{t}, W Y X_{k g g^{\prime} m m^{\prime} c c^{\prime}}^{t}, \\
& X X_{m c c^{\prime}}^{t}, Y X_{g m c}^{t}, Y Y_{g m m^{\prime}}^{t} \in\{0,1\} B A_{k g}^{t}, \\
& B Z_{k}^{t}, z, a_{k g}^{t} \geq 0 .
\end{aligned}
$$

\section{Computational experience}

In the following, the experiments conducted to evaluate the capability of solving the CMS problem through the presented model and applied approach are presented. Three instances were randomly generated. Experiments were performed on the implemented and solved model on a Core i5 PC with 1 GB of RAM using GAMS 23.5. The input data of generated instances are given in Tables 3-5. According to Bagheri and Bashiri [8], to obtain an optimal solution, two problems of CF and workforce assignment should be simultaneously solved. To this end, the present paper aims to separate and solve these two problems in three modes, namely the

Table 3. Numerical assumptions of different instances

\begin{tabular}{ccccccc}
\hline Instance & $\begin{array}{c}\text { Number of } \\
\text { machines }\end{array}$ & $\begin{array}{c}\text { Number of } \\
\text { parts }\end{array}$ & $\begin{array}{c}\text { Number of } \\
\text { time periods }\end{array}$ & $\begin{array}{c}\text { Number of } \\
\text { cells }\end{array}$ & $\begin{array}{c}\text { Number of } \\
\text { operators }\end{array}$ & $\begin{array}{c}\text { Number of } \\
\text { machine tools }\end{array}$ \\
\hline Instance 1 & 3 & 2 & 3 & 2 & 4 & 5 \\
Instance 2 & 4 & 4 & 3 & 2 & 6 & 7 \\
Instance 3 & 3 & 4 & 3 & 2 & 8 & 10 \\
\hline
\end{tabular}


Table 4. Numerical assumptions of different instances.

\begin{tabular}{cccccccc}
\hline $\begin{array}{c}\text { Machine } \\
\text { breakdown } \\
\text { rate }\end{array}$ & $\begin{array}{c}\text { Machine } \\
\text { repairing } \\
\text { cost }\end{array}$ & $\begin{array}{c}\text { Tool } \\
\text { consumption } \\
\text { cost }\end{array}$ & Inter-cell trips & $\begin{array}{c}\text { Demand } \\
\text { value }\end{array}$ & $\begin{array}{c}\text { Inter-cell } \\
\text { distances }\end{array}$ & $\begin{array}{c}\text { Machining time } \\
\text { per operation } \\
\text { (seconds) }\end{array}$ \\
\hline $\begin{array}{c}\text { (Integer } \\
\text { uniform) }\end{array}$ & $\begin{array}{c}\text { (Integer } \\
\text { uniform) }\end{array}$ & $\begin{array}{c}\text { (Integer } \\
\text { uniform) }\end{array}$ & Machines & Parts & $\begin{array}{c}\text { (Integer } \\
\text { uniform) }\end{array}$ & $\begin{array}{c}\text { (Integer } \\
\text { uniform) }\end{array}$ & $\begin{array}{c}\text { (Integer } \\
\text { uniform) }\end{array}$ \\
\hline$[0.01,0.05]$ & {$[35,37]$} & {$[10,17]$} & 20 & {$[12,16]$} & {$[10,14]$} & {$[3,5]$} & {$[0.09,0.11]$} \\
\hline
\end{tabular}

Table 5. Numerical assumptions of different instances.

\begin{tabular}{|c|c|c|c|c|c|c|c|c|}
\hline \multirow{2}{*}{$\begin{array}{c}\text { Minimum } \\
\text { number of } \\
\text { required } \\
\text { operators per } \\
\text { period } \\
\end{array}$} & \multicolumn{2}{|c|}{$\begin{array}{l}\text { Number of } \\
\text { machines in } \\
\text { each cell }\end{array}$} & \multicolumn{2}{|c|}{$\begin{array}{c}\text { Number of } \\
\text { tools on } \\
\text { each machine }\end{array}$} & \multicolumn{2}{|c|}{$\begin{array}{l}\text { Maximum time } \\
\text { capacity }\end{array}$} & \multirow[t]{2}{*}{$\begin{array}{l}\text { Learning } \\
\text { rate }\end{array}$} & \multirow[t]{2}{*}{$\begin{array}{l}\text { Forgetting } \\
\text { rate }\end{array}$} \\
\hline & $\operatorname{Max}$ & Min & $\operatorname{Max}$ & Min & Machine & Operator & & \\
\hline 3 & 4 & 1 & 4 & 2 & 50 & 11 & 0.015 & 0.005 \\
\hline
\end{tabular}

Table 6. A comparison of hierarchical and simultaneous approaches.

\begin{tabular}{ccccc}
\hline Instance & Model 1 & Model 2 & $\begin{array}{c}\text { Hierarchical } \\
\text { approach }\end{array}$ & $\begin{array}{c}\text { Simultaneous } \\
\text { approach }\end{array}$ \\
\hline Instance 1 & 3216 & 102 & 3319 & $\mathbf{3 3 1 9}$ \\
Instance 2 & 2884 & 166 & 3050 & $\mathbf{3 0 5 0}$ \\
Instance 3 & 1646 & 209 & 1855 & $\mathbf{1 7 9 6}$ \\
\hline
\end{tabular}

solo, hierarchical, and simultaneous modes. The two separated models are as presented below:

$\begin{array}{cc}\text { Model 1 (CF) } & \text { Model 2 (OS) } \\ \text { Min 3-8 } & \text { Min 9-11 } \\ \text { Subjected to: } & \text { Subjected to: } \\ 12-24,37 & 25-37\end{array}$

First, the generated instances are solved through Model 1. Then, the obtained results are introduced to Model 2 as input parameters; Model 2 is consequently implemented to solve the workforce assignment problem. The second model is also solved in isolation. Finally, the simultaneous model is implemented to compare the results. Table 6 reports the obtained results. As observed in Table 6, increasing the size of problem results in a better performance of the simultaneous model. Compared to the hierarchical mode, this model can reach more optimal solutions, especially in the case of large-scale problems. Moreover, the schematic view of Instance 3 is illustrated in Figure 1. Of note, this figure only demonstrates the solutions of periods 1 and 2 .

It is evident from Table 6 that the CF problem solution has a stronger effect on the final solution than that of the OS problem. Therefore, these two problems cannot be compared in such a condition. In order to overcome this obstacle, the LP-metric approach was implemented.

Generally, the LP-metric techniques prepare an extensive approach to solving MCDM problems while objectives are incommensurable and they are in contrast with each other. These techniques transform $m$ objectives (criteria) into one objective by utilizing the summation of normalized objectives; then, nondominated optimal answers could be achieved by utilizing the mentioned single-objective function. Normalizing is required as the objective (criterion) is incongruity dimension.

In fact, LP-metric can be used to achieve commensurate units of objective functions.

$$
D=\left(\sum_{i=1}^{n} \lambda_{i}\left(\frac{f_{i}^{*}-f_{i}(x)}{f_{i}^{*}-f_{i}^{-}}\right)^{p}\right)^{\frac{1}{p}}, \quad p=1,2, \ldots
$$

In the above equation, $f_{i}(x), f_{i}^{*}(x)$, and $f_{i}^{-}(x)$ are objective function, ideal objective function, and antiideal objective function, respectively. In addition, $\lambda_{i}$ is the weight given due the importance of the $f_{i}(x)$. 


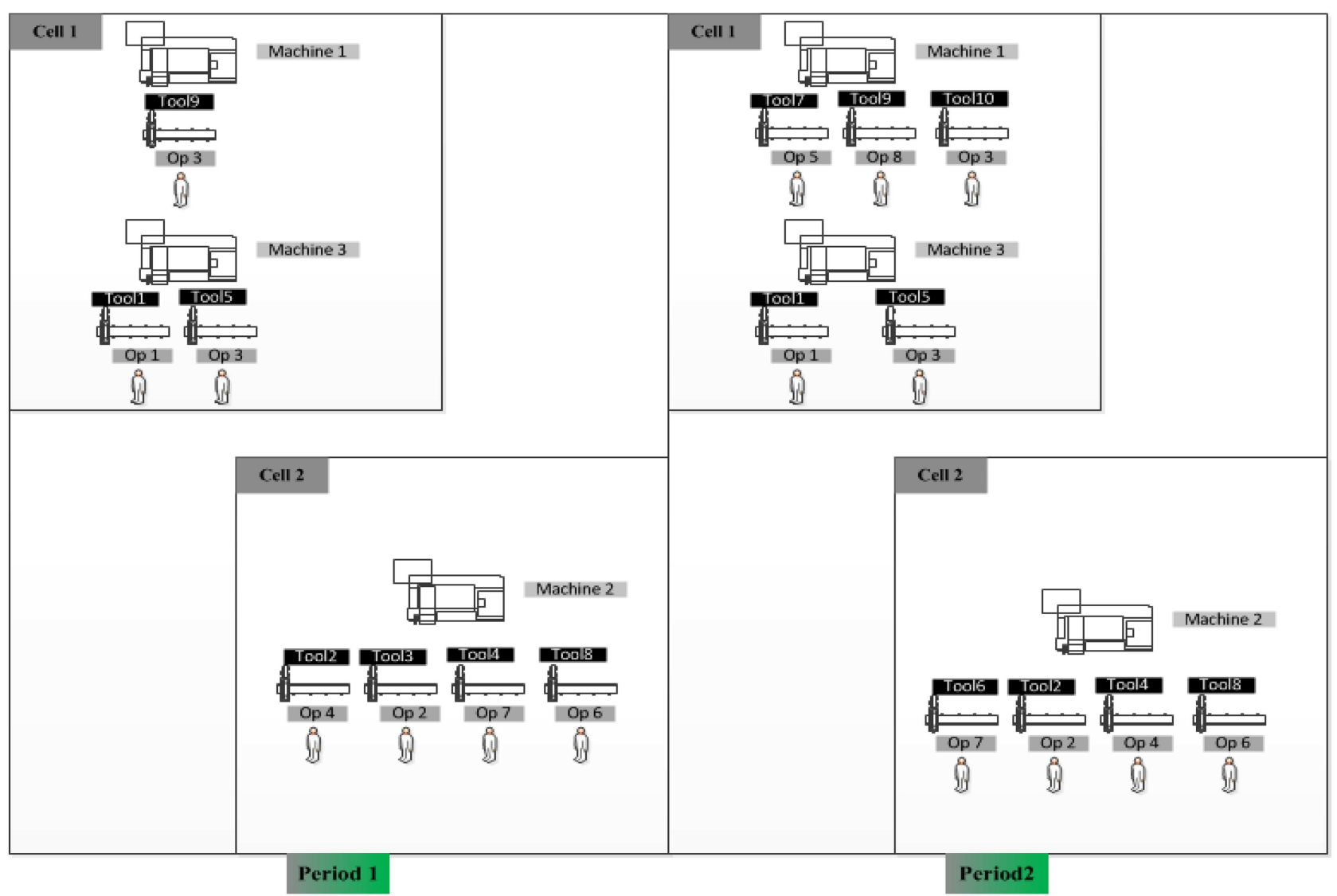

Figure 1. The schematic view of the solution to Instance 3.

Table 7. The trade-off matrix of Cell Formation (CF) and OS problems.

\begin{tabular}{ccccc}
\hline & \multicolumn{2}{c}{ Positive ideal solutions } & \multicolumn{2}{c}{ Negative ideal solutions } \\
\hline & $f_{1}$ & $f_{2}$ & $f_{1}$ & $f_{2}$ \\
\hline$f_{1}$ & $f_{1}^{*}=1569.979$ & 273 & $f_{1}^{-}=17968.82$ & 271 \\
$f_{2}$ & 13156.49 & $f_{2}^{*}=209.484$ & 10634.63 & $f_{2}^{-}=549.491$ \\
\hline
\end{tabular}

Attempts were made to minimize the distance between the ideal and anti-ideal pursuant to the constraints and discover the non-dominated solution. Therefore, in Eq. (118), LP-metric represents the distance between $F(x)$ and $F^{*}(x)$.

Based on this approach, the trade-off matrix can be generated, as shown in Table 7 .

By changing the $\lambda_{i}$ value, which represents the weight considered for each of the aforementioned problems, the Pareto optimal solution can be obtained, as shown in Figure 2.

As observed in this figure, workforce-related costs have a significant impact on the CF solution. In order to analyze the OS problem in detail, expert workforce who worked only with a machine tool without any training cost was carefully supervised. The salary of this workforce was naturally higher than that of others. In this situation and based on the results, workforce

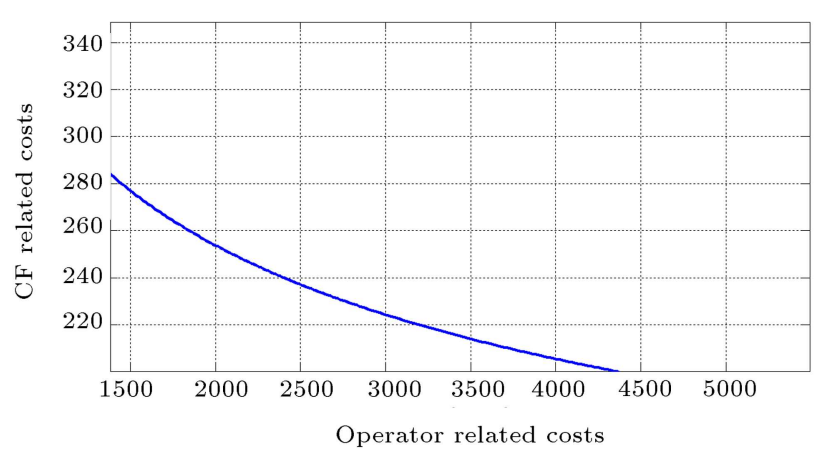

Figure 2. The pareto solution to Cell Formation (CF) and OS problems.

with a lower skill level was selected to be trained how to work with that machine tool. With a decrease in the salary of the expert workforce, he/she was selected as a machine tool workforce. 


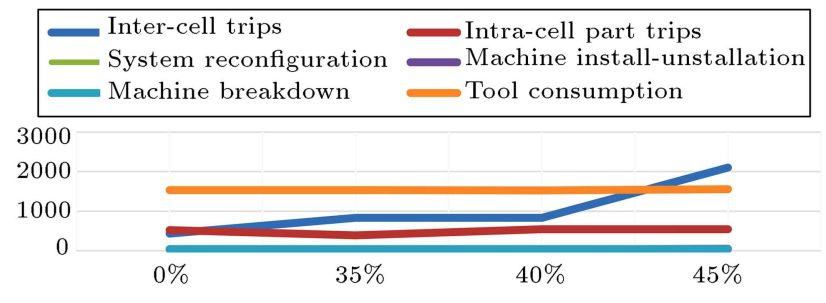

Figure 3. Cost sensitivity analysis versus demand rate.

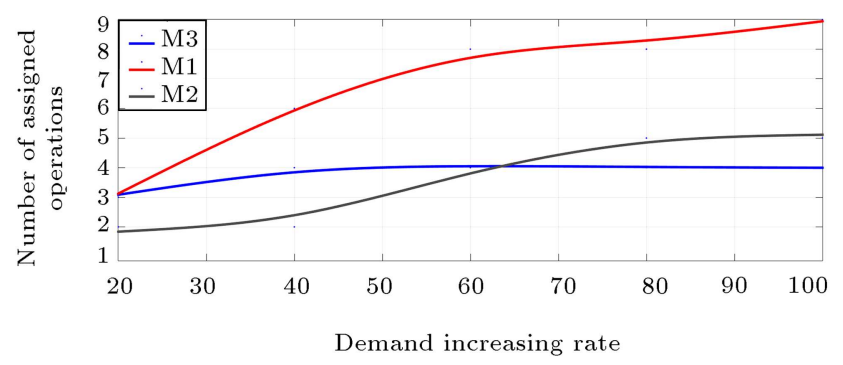

Figure 4. The increasing rate of demand vs. the number of operations assigned to a machine (period 1) Instance).

In addition, it can be concluded that any change in the demand value might significantly affect the essential cost terms, shown in Figure 3. According to this Figure, with an increase in the demand value, the inter-cell part trip cost has exponentially increased. Hence, a decision-maker should control the demand value to avoid extra inter-cell part trip costs. Moreover, a meticulous maintenance plan can decrease the rate of machine breakdown in high-demand companies.

Machines are the second main manufacturing resources required to be analyzed in detail. Machine breakdown and demand rate are of considerable significance in machine implementation in a manufacturing company. Figure 4 illustrates the increasing rate of demand on the total number of operations assigned to a machine in a production period (Instance 1 ).

According to this Figure 4, Machine 3 can process a maximum number of 4 operations in a production period. As mentioned earlier, a machine cannot be interrupted while processing a task. According to the input data, the rate of breakdown in machine 3 is higher than that in the other two machines. Therefore, it is preferable that this machine would process only 3 operations, even in a high-demand situation.

To adjust real-world cellular production system models, it is required to add more variables and limitations to the model, which will demand a lot of time to solve such models by time, memory, and processing power. As a result, nowadays, modern methods are applied to a Genetic Algorithm (GA). A $\mathrm{GA}$ is part of random search techniques that are used to solve NP-complete problems, such as cell-system production models.

In this section, MATLAB software (GA toolGenetic algorithm GUI) was utilized to solve the complexities of the hierarchical and simultaneous model with the GA to evaluate the performance of the models in vaster dimensions. In Table 8 , the dimensions of 4 numerical instances are solved using GA. The obtained results are given in both Table 9 and Figure 5. Of note, the simulation model has achieved a better result than the hierarchical model. By developing a variety of the dimensions of the models, the deviation of the optimum values obtained in the two models becomes more significant than ever.

Due to the lack of a similar article and a real case study in Iran, the sensitivity analysis of one of the examined examples mentioned in the paper was performed to validate the model. For instance, in Instance 4 , by assuming that the dimensions of the model were

Table 8. Numerical assumptions of instance.

\begin{tabular}{ccccccc}
\hline Instance & $\begin{array}{c}\text { Number of } \\
\text { machines }\end{array}$ & $\begin{array}{c}\text { Number of } \\
\text { parts }\end{array}$ & $\begin{array}{c}\text { Number of } \\
\text { time periods }\end{array}$ & $\begin{array}{c}\text { Number of } \\
\text { cells }\end{array}$ & $\begin{array}{c}\text { Number of } \\
\text { operators }\end{array}$ & $\begin{array}{c}\text { Number of } \\
\text { machine tools }\end{array}$ \\
\hline Instance GA 1 & 10 & 20 & 3 & 2 & 13 & 2 \\
Instance GA 2 & 15 & 25 & 3 & 3 & 14 & 3 \\
Instance GA 3 & 20 & 27 & 3 & 3 & 15 & 5 \\
Instance GA 4 & 25 & 30 & 3 & 5 & 16 & 6 \\
\hline
\end{tabular}

Table 9. A comparison of hierarchical and simultaneous approaches (genetic algorithm).

\begin{tabular}{ccccc}
\hline Instance & Model 1 & Model 2 & $\begin{array}{c}\text { Hierarchical } \\
\text { approach }\end{array}$ & $\begin{array}{c}\text { Simultaneous } \\
\text { approach }\end{array}$ \\
\hline Instance GA 1 & 7560 & 1003 & 8563 & $\mathbf{8 2 9 4}$ \\
Instance GA 2 & 8640 & 2845 & 11485 & $\mathbf{9 8 6 9}$ \\
Instance GA 3 & 10380 & 5004 & 15384 & $\mathbf{1 0 3 8 4}$ \\
Instance GA 4 & 15230 & 9384 & 24614 & $\mathbf{1 7 9 7 4}$ \\
\hline
\end{tabular}




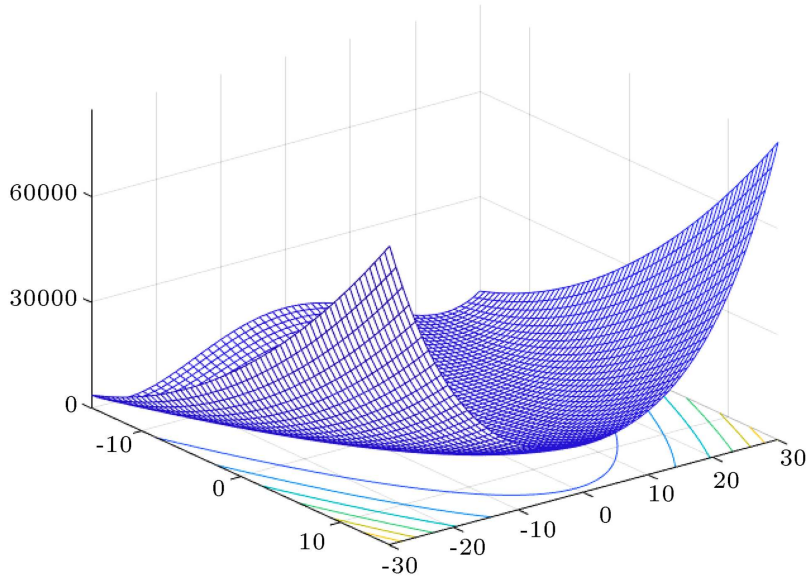

Figure 5. Functional behavior in the genetic algorithm.

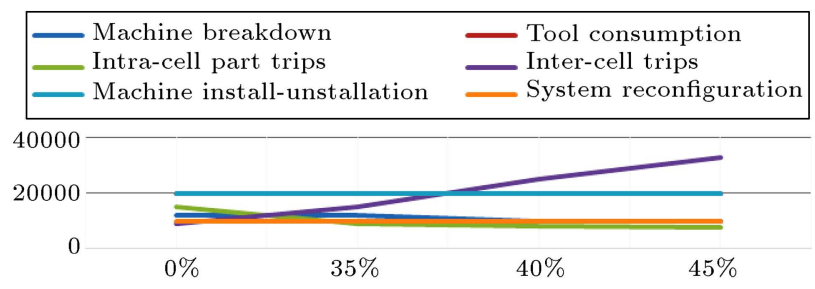

Figure 6. Cost sensitivity analysis versus demand rate in the genetic algorithm.

constant, the sensitivity of the model parameters was analyzed. For example, the sensitivity analysis of the demand parameter that changed all decision variables and optimal values is shown in Figure 6. Some quantities were not significantly different in costs such as the cost of installing machines and cellular configurations. In other cases, the breakdown of machines and cost of intercellular mobility were considerably high; therefore, the resulting changes seem reasonable.

\section{Conclusion}

The present paper proposed a new-fangled model to design an efficient Cellular Manufacturing System (CMS). The basic assumptions of the proposed model were the incorporation of machine tools, machine breakdown, and the workforce learning-forgetting effect. To the best of the authors' knowledge, only a few researchers have focused on such real-world parameters. In terms of both computational time and optimality, the experimental results verified the efficiency of the proposed approach. Moreover, analytical experiments were performed to assess the sensitivity of the presented model and it could be concluded that the machine failure played a key role in elevating the performance of CMS, especially in companies of high demands. In addition, workforce-related costs were found to have a strong impact on the cell formation solution. Another sensitivity analysis of the proposed model revealed the impact of changing demand on the rate of machine utilization. In other words, by increasing the demand value, the machine with the minimum breakdown cost value was implemented more often than other ones. Besides, nowadays, in the competitive atmosphere of the world, the workforce represents the main production resources. Hence, analyzing and proposing new models is essential to optimally solve OS problems. In this paper, some workforcerelated issues including hiring, firing, their salary, training, and the workforces' learning-forgetting effect were taken into account. Given all these considerations, the developed mathematical model could be employed for factories with the capability of having a cellular design. In fact, numerous industrial factories such as car manufacturers with a rich diversity in their product types and fluctuations in the demand value can employ the proposed model with the aim of designing an optimal CMS via the minimum amount of costs. The main objective of this research was to consider some real-world production elements to be applied to many factories.

As a guideline for future studies, it would be motivating to develop some solution approaches to optimally solve the model. Moreover, incorporating other real-world industrial factors such as intra-cell GL and machine duplication in providing a framework can be of great value for future research. Additionally, the concept of uncertainty can be considered in the provided framework. As an instance, uncertainty in the demand value or the processing time is one of the main issues in real world application and thus, it can be explored in more detail in future studies.

\section{Acknowledgement}

The authors would like to state their gratitude to Iran National Science Foundation (INSF) for all their kind support of the present project.

\section{References}

1. Liu, C., Wang, J., and Leung, J.Y.T. "Integrated bacteria foraging algorithm for cellular manufacturing in supply chain considering facility transfer and production planning", Applied Soft Computing, 62, pp. 602-618 (2018).

2. Askin, R.G. "Contributions to the design and analysis of cellular manufacturing systems", International Journal of Production Research, 51, pp. 6778-6787 (2013).

3. Ameli, M.S.J. and Arkat, J. "Cell formation with alternative process routings and machine reliability consideration", The International Journal of Advanced Manufacturing Technology, 35, pp. 761-768 (2008).

4. Bulgak, A.A. and Bektas T. "Integrated cellular manufacturing systems design with production planning and 
dynamic system reconfiguration", European Journal of Operational Research, 192, pp. 414-428 (2009).

5. Mehdizadeh, E., Niaki S.V.D., and Rahimi, V. "A vibration damping optimization algorithm for solving a new multi-objective dynamic cell formation problem with workers training", Computers \& Industrial Engineering, 101, pp. 35-52 (2016).

6. Mahdavi, I., Aalaei, A., Paydar, M.M., and Solimanpur, M. "Designing a mathematical model for dynamic cellular manufacturing systems considering production planning and worker assignment", Computers \& Mathematics with Applications, 60, pp. 1014-1025 (2010).

7. Aryanezhad, M.B., Deljoo, V., and Mirzapour Al-eHashem, S. "Dynamic cell formation and the worker assignment problem: a new model", The International Journal of Advanced Manufacturing Technology, 41, pp. 329-342 (2009).

8. Bagheri, M. and Bashiri M. "A new mathematical model towards the integration of cell formation with workforce assignment and inter-cell layout problems in a dynamic environment", Applied Mathematical Modelling, 38, pp. 1237-1254 (2014).

9. Javadi, B., Jolai, F., Slomp, J., Rabbani, M., and Tavakkoli- Moghaddam, R. "An integrated approach for the cell formation and layout design in cellular manufacturing systems", International Journal of Production Research, 51, pp. 6017-6044 (2013).

10. Bagheri, M., Sadeghi, S., and Saidi-Mehrabad, M. "A benders' decomposition approach for dynamic cellular manufacturing system in the presence of unreliable machines", Journal of Optimization in Industrial Engineering, 17, pp. 37-49 (2015).

11. Bayram, H. and Şahin, R. "A comprehensive mathematical model for dynamic cellular manufacturing system design and linear programming embedded hybrid solution techniques", Computers \& Industrial Engineering, 91, pp. 10-29 (2016).

12. Azadeh, A., Ravanbakhsh, M., Rezaei-Malek, M., Sheikhalishahi, M., and Taheri-Moghaddam, A. "Unique NSGA-II and MOPSO algorithms for improved dynamic cellular manufacturing systems considering human factors", Applied Mathematical Modelling, 48, pp. 655-672 (2017).

13. Liu, C., Wang, J., and Leung, J.Y.T. "Worker assignment and production planning with learning and forgetting in manufacturing cells by hybrid bacteria foraging algorithm", Computers \& Industrial Engineering, 96, pp. 162-179 (2016).

14. Mehdizadeh, E. and Rahimi, V. "An integrated mathematical model for solving dynamic cell formation problem considering workforce assignment and inter/intra cell layouts", Applied Soft Computing, 42, pp. 325-341 (2016).

15. Rafiei, H. and Ghodsi, R. "A bi-objective mathematical model toward dynamic cell formation considering labor utilization", Applied Mathematical Modelling, 37, pp. 2308-2316 (2013).

16. Sakhaii, M., Tavakkoli-Moghaddam, R., Bagheri, M., and Vatani, B. "A robust optimization approach for an integrated dynamic cellular manufacturing system and production planning with unreliable machines", $A p$ plied Mathematical Modelling, 40, pp. 169-191 (2016).

17. Nouri, H. "Development of a comprehensive model and BFO algorithm for a dynamic cellular manufacturing system", Applied Mathematical Modelling, 40, pp. 1514-1531 (2016).

\section{Biographies}

Majid Rafiee received his BSc degree in Industrial Engineering from Isfahan University, Isfahan, Iran in 2003 and his MSc and PhD degrees in Industrial Engineering from Sharif University of Technology, Tehran, Iran in 2006 and 2013, respectively. In 2013, he joined the Department of Industrial Engineering at Sharif University of Technology, Tehran, Iran as an Assistant Professor. Dr. Rafiee's research interests include stochastic programming, operations research, mathematical programming, quality management, and quality control.

Atye Mohammaditalab received his BSc degree in Industrial Engineering from Sharif university of Technology, Tehran, Iran, and his MSc degree in Mathematics from University of Tehran, Tehran, Iran in 2013 and 2016, respectively. Her main areas of interest are integer programming, mathematical modeling, and optimization. 